\title{
On the question of quantitative modeling of the process of freezing products
}

\author{
Evgeniy Semenov ${ }^{1}$, Yury Sidorenko $^{1}$, Svetlana Tefikova ${ }^{1}{ }^{*}$, Mikhail Sidorenko $^{2}$, \\ Sergey Shterman ${ }^{2}$ \\ ${ }^{1}$ K.G. Razumovsky Moscow State University of technologies and management \\ (The First Cossack University), St. Zemlyanoy Val, 73, Moscow, Russian Federation \\ ${ }^{2}$ GEON LLC, Obolensk, Obolenskoe sh., str. 1, Moscow region, Russia
}

\begin{abstract}
It is known that the cooling of food prevents the reproduction of microflora, thus contributing to the preservation of the beneficial properties of products. Despite this, the cooling process is still the least studied stage of cold processing, which necessitates further in-depth study of the problem discussed in the article. Given the diversity and volume of processing facilities and the energy resources they consume, it is important to justify the analytical and numerical apparatus to address many engineering and plant-based cooling products of technological challenges. Below, in the article, computer-based tools are used to be different from the method used conveniently enough for practical purposes to quantify and calculate the common in production process of food freezing.
\end{abstract}

\section{Introduction}

It is known that, along with the problem of cooling plant-based substances, while achieving a reduction in energy costs, a similar problem is forced to solve, in general, and the entire human community in a wide variety of tasks to justify the most rational technologies in the field of organic and inorganic systems. Where does the importance of the research carried out in the cooling technology of various substances, both theoretically and experimentally, arise [1-9]. Since in some cases heat transfer takes place in objects with a complex, greatly complicating problem of physical and mathematical formalization of the studied phenomenon geometric structure, researchers as imitating the form of a real body have to choose one or more surfaces of the canonical species, usually a plane, a cylinder or a sphere.

This paper theoretically explores the process of freezing a ball-shaped product such as a test blank. The task of forming frozen and cooled areas of the body in question is interpreted as a complicated (due to phase transition) change in the aggregate state of the environment, the problem of heat exchange between the cooled zone I and the frozen zone II (Fig. 1).

\footnotetext{
* Corresponding author: s.tefikova@mgutm.ru
} 


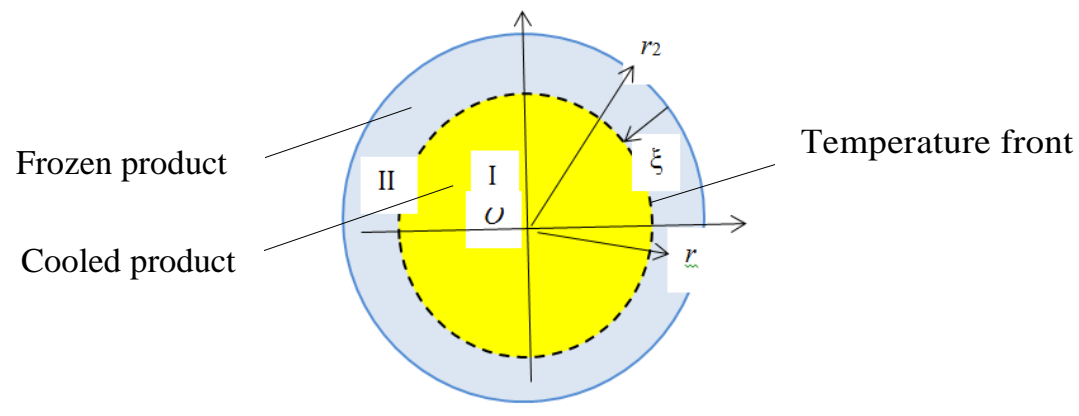

Fig. 1. Scheme to calculate the freezing process of the test blank ( $r$ - current radial coordinate, $\mathrm{r} 2$ - the radius of the blank, $\xi$ - the coordinate of the temperature front)

Since heat-physical ratios at the boundaries of the environment are supposed to change in leaps and bounds, additional heat is required at the crossings. The problem is solving on the basis of the model of the paired task of the cooling of the heat transfer task of Stefan for two zones of product I and II in two different aggregate states. At the same time, on the basis of the developed algorithm, the quantitative dependence of the coordinates of the temperature front on the time of the freezing process of the preparation is justified, at different values of temperature indicators on the boundaries of zones and the content of the product.

In the accepted assumption that the cooled product is geometrically modeled by the ball, as limiting zones I and II surfaces choose the sphere.

Since Stefan's task is non-linear in the overall setting, it is not analytically solvable, so that approximate methods are used for her research.

\section{Material and methods}

It is assumed that in the refrigeration chamber with a constant temperature $\mathrm{T}_{2}$ suddenly placed modeling frozen cooled product homogeneous spherical body radius $r_{2}$ (Fig. 1). In the future, to analyze the dynamics of the formation of the freezing zone in the ball, preliminarily, decide the system of differential equations of thermal conductivity for each of the zones I and II. After that, based on the received ratios on the temperature profile in the zones and the structure of the differential equation of the first order, Stefan's condition on heat exchange at the front of the temperature $\xi=\xi(t)$ calculated the dependence of the coordinates of the temperature front on the time of the process of freezing the ball harvest.

Inside each of the frozen and cooled zones I and II, the temperature of the environment in spherical coordinates satisfies the differential equations of thermal conductivity and the boundary conditions of the first kind (Fig. 1)

$$
\begin{gathered}
\partial u_{1} / \partial \tau=a_{1}\left(1 / r^{2}\right) \partial\left(r^{2} \partial u_{1} / \partial r\right) / \partial r \quad\left(\tau>0, r_{1}<r<\xi\right), \\
\partial u_{2} / \partial \tau=a_{2}\left(1 / r^{2}\right) \partial\left(r^{2} \partial u_{2} / \partial r\right) / \partial r \quad\left(\tau>0, \quad \xi<r<r_{2}\right), \\
u_{1}\left(r_{1}, \tau\right)=T_{1}, \\
u_{1}(\xi, \tau)=\mathrm{T}_{3}, \\
u_{2}(\xi, \tau)=T_{3}, \\
u_{2}\left(r_{2}, \tau\right)=\mathrm{T}_{2}
\end{gathered}
$$

where $\tau$ - time; $u_{1}, u_{2}, u_{3}$ - respectively, temperature in zone I, zone II and on the temperature front; $a_{1}, a_{2}$-temperature ratios for areas I and II (Fig. 1 area is not shown). 
If it is believed that on the border $\xi=\xi(\tau)$ the supply of heat from zone I and from the side of zone II is implemented by the law of Fourier's heat transmission, then in the assumption that the density of the frozen and cooled areas of the product is approximately the same $\left(\rho_{1} \approx \rho_{2}=\rho\right)$ on the moving boundary of freezing is true condition of Stefan

$$
\left.\left[\frac{\partial\left(\lambda_{1} u_{1}-\lambda_{2} u_{2}\right)}{\partial r}\right]\right|_{r=\xi(\tau)}=m \rho \zeta \frac{d \xi}{d \tau}
$$

where $\lambda_{1}, \lambda_{2}$ - respectively, the thermal conductivity factor for areas I and II; $m$ - in-water content of the product, $\zeta$ - the hidden heat of the phase transition of water to the ice.

Then come to a system of three joint differential equations (1), (2), (7) relative to the required values $u_{1}, u_{2}, \xi$, whose solution is generally difficult. If the temperature difference in the zones is small and the process of heat transfer develops for a long time, the mode of heat transfer in zones I and II can be relied on quasi-stationary. In this case, the equations (1), (2) are written in the form of:

$$
\begin{array}{ll}
\frac{\partial\left(r^{2} \partial u_{1} / \partial r\right)}{\partial r}=0 & \left(\tau>0, r_{1}<r<\xi\right), \\
\frac{\partial\left(r^{2} \partial u_{2} / \partial r\right)}{\partial r}=0 & \left(\tau>0, \xi<r<r_{2}\right),
\end{array}
$$

(3) - (6) is a decision that is consistent with the terms and conditions

$$
\begin{array}{ll}
u_{1}(r, \tau)=T_{1}+\frac{\xi\left(r-r_{1}\right)\left(T_{1}-T_{3}\right)}{r\left(r_{1}-\xi\right)} & \left(\tau>0, r_{1}<r<\xi\right), \\
u_{2}(r, \tau)=T_{2}+\frac{\xi\left(r-r_{2}\right)\left(T_{3}-T_{2}\right)}{r\left(\xi-r_{2}\right)} & \left(\tau>0, \xi<r<r_{2}\right) .
\end{array}
$$

Then in effect (7)-(9) get

$$
\frac{\lambda_{1} r_{1}\left(\xi-r_{2}\right)\left(T_{1}-T_{3}\right)-\lambda_{2} r_{2}\left(r_{1}-\xi\right)\left(T_{3}-T_{2}\right)}{\left(r_{1}-\xi\right)\left(\xi-r_{2}\right)}=m \rho \zeta \xi \frac{d \xi}{d \tau} .
$$

Ordinary first-order differential equation (10) relative $\xi$ deciding to reconcile it with the initial condition

$$
\xi=0 \text { at } \tau=0 .
$$

Solution to the problem (10), (11) is

$$
\tau=m \rho \zeta \cdot \int_{\xi}^{r_{2}} \frac{\left(r_{1}-\xi\right)\left(\xi-r_{2}\right) \xi d \xi}{\lambda_{1} r_{1}\left(\xi-r_{2}\right)\left(T_{1}-T_{3}\right)-\lambda_{2} r_{2}\left(r_{1}-\xi\right)\left(T_{3}-T_{2}\right)}
$$

Since the equation (12) contains the resulting value - the variable $\xi$-implicitly, this ratio is resolved relatively $\xi$ using the standard root procedure of the Mathcad computer environment. 


\section{Results and discussion}

The quantitative simulation of the process of freezing the product of the spherical shape by dependency (12) was carried out on the basis of the following physical-mechanical and geometric parameters of the processing process: the size of the ball: $r_{1}=0, r_{2}=0,06 \mathrm{~m}$ (i.e.. as the object of the study choose the body in the form of a solid ball); the temperature, respectively, on the surface of the body $T_{1}=293 \mathrm{~K}$, placed in the cooling environment, the phase transition of water into the ice $T_{3}=272 \mathrm{~K}$, on the outer boundary of the body temperature attached to the values of $T_{2}=253$ and $263 \mathrm{~K}$, thermal conductivity ratio for areas I (water) and II (ice), respectively, $\lambda_{1}=0,6 ; \lambda_{2}=2,3 \mathrm{~W} /(\mathrm{m} \cdot \mathrm{K})$; in-content of the product $m=0,5 ; 0,7$; Hidden heat of phase water transition into ice $\zeta=0,33 \times 10^{6} \mathrm{~J} / \mathrm{kg}$; body density $\rho=1100 \mathrm{~kg} / \mathrm{m}^{3}$.

Which, according to the parameters of the study, in aggregate. typical, for example, for a product such as a test preparation.

Visualization of the data of the numerical experiment shows a natural exponential relatively slow increase in the time of processing of the product cold to the temperature of the cooling environment. That is, on the one hand, the assumption about the quasistationary nature of the process of heat transfer is confirmed. On the other hand, the graphics reflected are Fig. 2 results of the calculation reveal the consent of the calculation results with the physical meaning of the phenomenon under study. Thus, with all other fixed parameters, a large difference in the temperature of the external environment and the cooled body initiates its faster cooling (Fig. 2 of the temperature front graph 1 above schedule 2). In turn, greater body water content naturally reduces the effect of the transfer of heat to the cooled body, as the thermal conductivity ratio of the ice is higher than that of the water (Fig. 2 of the temperature front graph 1 above schedule 3).

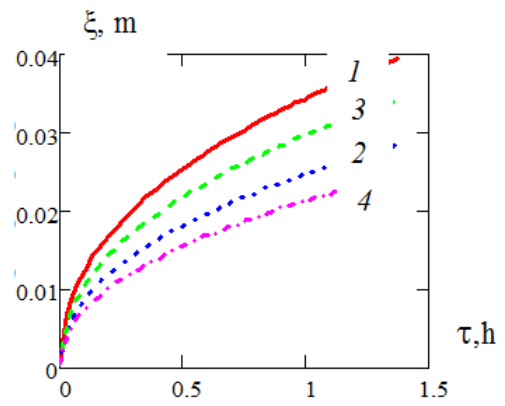

Fig. 2. Dependences of the coordinates $\xi$ the front of the freezing from time $\tau$ the process of freezing the ball harvest at the temperature of $T_{1}=293 \mathrm{~K}$ in the center of the $O$ and temperature of $T_{3}=272 \mathrm{~K}$ to the phase transition at the front of $r=\xi$ at different values of $T_{2}$ temperature on the surface $r=r_{2}$ and the ice-holding $m$ in the blank ( $m=0,5: 1-T_{2}=$ $\left.253,2-T_{2}=263 \mathrm{~K} ; m=0,7: 3-T_{2}=253,4-T_{2}=263 \mathrm{~K}\right)$

\section{Conclusions}

Thus, within the framework of the restrictions and conditions for changing the aggregate state, an important effective and simple way to quantify the process of transferring heat to a ball-shaped solid body has been developed in the context of the set of the task. That simulates the dynamics of the temperature field in processing time when the product of this kind is frozen. It is obvious that the study can be developed in conditions of unsteady cold transport or when the body is treated with cold under the influence of a high-intensity temperature gradient. 


\section{References}

1. A.V. Lykov, Heat conduction theory, (M.: Vysshaya shkola, 1967)

2. L.I. Rubinstein The Stefan problem, (Riga: Znajzne, 1967)

3. A.M. Br azhnikov, V.A. Karpychev, A.I. Peleev Analytical methods for studying the processes of heat treatment of meat products, (Moscow: Food industry, 1974)

4. E.V. Semenov, V.A. Karamzin, A.V. Karamzin Methods of calculating technological processes of mass and heat transfer in processing industries of the agro-industrial complex, (Moscow: Sputnik + Company, 2009)

5. V.V. Ostapenko, V.V. Lukyanov, V.V. Bulletin of the Voronezh State Technical University. 10, 5, 89 (2014)

6. K. Zhichkin, V. Nosov, L. Zhichkina, O. Grigoryeva, V. Kondak, T. Lysova, IOP Conf. Series: Earth and Environmental Science, 012004 (2020)

7. V.V. Nosov, O.K. Kotar, M.M. Kosheleva, L.N. Alaikina, N.A. Novikova, Ecology, Environment and Conservation. 20, 4, 1857 (2014)

8. V.M. Arapov, D.A. Kazartsev, I.A. Nikitin, M.V. Babaeva, S.V. Zhukovskaya, S.N. Tefikova, G.V. Posnova, I.V., International Journal of Advanced Computer Science and Applications. 11 (2), 317-322 (2020)

9. A.A. Kostoglotov, S.V. Lazarenko, I.A. Nikitin, XIV Int. Scientific-Technical Conf. "Dynamic of Technical Systems" (DTS-2018). 226, 04031 (2018) 